rise of temperature in acute eczema, if present at all, is only trifling, possibly a degree, and the patient who looks so ill feels quite well, though perhaps tormented with itching.

There is, however, another, the relapsing, form of erysipelas, to which I have given the descriptive name of relapsing streptococcal lymphangitis. This is in reality a chronic disease with acute exacerbations. The patient will generally give a history somewhat of this sort. At first there was an ordinary attack of acute erysipelas, with fever and illness, and this got well. After a variable period a second attack ensued over the same area, accompanied by less serious constitutional symptoms, and when it disappeared perhaps some slight thickening of the affected parts was left behind. Later, many attacks occurred, the intervening periods of health becoming progressively shorter and all constitutional symptoms remaining absent.

In these cases the sharp margin and defined outline are lost, bullæ do not arise, but the whole eruption is limited to a somewhat red and shining œdema with some permanent thickening. The

\section{THE PROPHYLAXIS OF SCARLET FEVER AND DIPHTHERIA.*}

BY

\author{
DAVID C. KIRKHOPE, M.D., D.P.H.,
} MEDICAL OFFICER OF HEALTH FOR TOTTENHAM, N.

I HAVE chosen this subject for consideration because I have come to the conclusion that we are on the eve of a revolution respecting the methods by which these diseases will be controlled.

If it was ever anticipated that isolation of the infectious sick in infectious diseases hospitals would result in the suppression of the maladies concerned, I think it will be conceded generally that those hopes have not been realised. Not only is this so, but there has been the disadvantage in these hospitals of cross infections with accompanying complications and mortality. These disadvantages are not negligible, for, quoting from the report of the Metropolitan Asylums Board for the year 1922 (the last year for which similar information is available), it is shown that 15,279 scarlet fever cases were admitted to the Metropolitan Asylums Board's hospitals and of these-

$\begin{array}{rrrr}1826 & \text { contracted one other disease, } \\ 118 & \text {, } & \text { two , diseases, } \\ 6 & \text { " } & \text { three , } & , \\ 2 & \text {, } & \text { four , } & ,\end{array}$

i.e., $12 \cdot 77$ per cent. of the cases contracted at

* A Lecture delivered before the North-East London PostGraduate College on Jan. 21 st, 1927. diagnosis is made on the permanent thickening, की the absence of the true eczematous signs, and the history of frequent relapse with serious fever and $\cong$ illness at the first one or two attacks. Such $\frac{\mathbb{\Phi}}{3}$ patients have nearly always a fissure somewhere in the vestibule of the nose, most commonly either ${ }^{\complement}$ at the anterior angle of the nasal orifice or at the $\overrightarrow{\vec{s}}$. junction of the ala nasi with the cheek. This healsfrom time to time, generally remaining slightly등 red, especially in cold weather. On the patient $\bar{c}$. developing a coryza it opens again and the strepto- $\widehat{\Phi}$ coccal lymphangitis recurs. Constitutional immunity has developed so far that the patient no longeres feels very ill, though perhaps slightly out of sorts, $\overrightarrow{0}$ and the main discomfort remains local. I regardthis as a very interesting condition, because it is $\bar{\sigma}_{\sigma}$ so good an example of the importance of generato constitutional immunity as contrasted with local tissue immunity. I have worked at it carefully. by means of attempted cultivation and inoculation, N but have been unsuccessful, and this is perhaps not surprising in view of the comparatively high $\vec{\infty}$ state of constitutional resistance which is present.응

(To be concluded.)

least one other disease, and 48 deaths occurred amongst them.

Similarly with regard to diphtheria, 11,737 cases were admitted in 1922 , of whom 1207 contracted other diseases-viz., 10.28 per cent. Of these, 1136 contracted one other disease, 68 contracted two other diseases, 3 contracted three other diseases, and of these 79 died.

In the four years 1922-25 inclusive, 4967 cases were admitted under a wrong diagnosis of scarletō fever and 72 of them died, and in the same period ${ }^{3}$ 8634 wrongly diagnosed as diphtheria were admitted with 198 deaths.

\section{Scarlet Fever.}

The type of scarlet fever has now so greatly윽 altered from that prevailing 20 years ago that $a$ fatal case is rare, and the Metropolitan Asylums? Board, towards the end of last year, resolved that as scarlet fever was now such a mild ailment as은 compared with measles they had determined to $>$ give precedence to cases of measles seeking admission to their institutions. The mildness of the type of scarlet fever now prevailing may be gauged from the fact that no death has occurred in Tottenham from that cause since November, 1923. It isc incumbent on those who have the administration of health services to prepare for the contingencyo of having to treat notified cases of scarlet fevero elsewhere than in isolation hospitals. Where there? is sufficient domestic accommodation there willo be no difficulty ; indeed, that is even now the mosto satisfactory way of dealing with the prevailing? mild type of the disease.

It happens opportunely that investigationso have been proceeding apace in America, and the researches of the Dicks have resulted in theo identification of an organism, or series of organisms, 
of the hæmolytic streptococcus type as the cause or causes of the specific disease known as scarlet fever. The Streptococcus hromolyticus complies with all Koch's postulates save one. It has not yet been proved that the disease caused by the organism is transmissible from one person to another. Dr. O'Brien, of the Wellcome laboratories, points out that this fact has somewhat greater weight than mere punctiliousness, for an analogous state of things occurred in the case of swine fever. The Bacillus suipestifer was regarded as the cause until it was discovered that the swine fever resulting from the inoculation with the $B$. suipestifer was not communicable. It now appears that $B$. suipestifer accounts for the symptoms, but the infectiousness of the disease is due to another organism--I believe a filter-passing organism with which the $B$. suipestifer is usually associated in the disease.

Assuming that that postulate can be satisfied we shall be in a fair way to cope with the epidemicity of the disease. What are the evidences upon which the assumption is based that the $S$. hoemolyticus is the specific organism? (1) It has been isolated from the throats of patients suffering from the disease. (2) Cultures swabbed on the throats of susceptible persons have caused a disease similar in all respects to scarlet fever. (3) The organism has been recovered from those patients. (4) A culture grown on bouillon has produced a toxin, a small quantity of which introduced intradermally into susceptible persons has produced a local reaction-the Dick test. (A patient recovered from scarlet fever does not respond in the same manner.) (5) An antitoxin has been produced in the horse; this antitoxin, mixed in appropriate proportion with the toxin, negatives the Dick test, and, administered in large doses, has a markedly modifying effect on cases acutely ill from scarlet fever. (6) A small intradermal injection of the antitoxin has the effect in a period of 10 to 15 hours of blanching the rash of scarlet fever-the SchultzCharlton reaction-an accurate means of verifying the diagnosis of scarlet fever. The antitoxin has no such effect on other rashes. Birkhaug, studying this Schultz-Charlton reaction, and using Krogh's method of examining the capillaries in situ, found that in the erythematous area under a 300 magnification, about as many as 40 loops could be counted in a single field. After the intradermal infiltration of 1 c.cm. of antitoxin from hour to hour the capillary loops diminish in diameter and the flow is retarded, so that when the SchultzCharlton phenomenon is complete the normal number of loops, 8 to 10 , are discernible. The early administration of antitoxin in scarlet fever by neutralising the toxin already in circulation is likely to cut short the illness and by so doing restrict the infective period of the case. Copious desquamation is not usual in those cases where antitoxin has been given early, as it must be if it is to be of value. It is probable that the toxins are so fixed in the tissue within the first two or three days that later treatment by antitoxin fails to neutralise them.

Birkhaug further found that the average duration of the S. scurlatince in the tonsillar crypts of clinical cases was 10 days. The longest period was 246 days from the onset. The disappearance of the organism from the crypts coincided with the time when the patient's blood had sufficient antitoxin to produce the Schultz-Charlton reaction in the rash of an acute case of scarlet fever.

The average duration of the infectivity of scarlet fever is a matter of some importance, from the point of view of an experiment I attempted two years ago in an infants' school, where scarlet fever had broken out. I got the consent of about 50 per cent. of the school contacts to inoculate them with anti-scarlet fever serum. I then spaced the children out alternately with the uninoculated. For a fortnight thereafter there were no further notifications and afterwards the cases notified were amongst those who had not been protected; 131 children were thus passively immunised with 5 c.cm. concentrated serum each.

The protection thus afforded lasts, however, about a fortnight only. This I was able to gauge not from school but from home contacts. In two instances I inoculated the contacts of a patient treated at home. Isolation was not attempted. The mother was not inoculated in either case. She contracted the disease, and the contacts who were protected against the primary case were unable to resist the infection of the extended period covered by the mother's illness. Indeed, as the passive immunity wore off, the children appeared to become more sensitive to the infection and went down with mild attacks one after the other.

A dose of 5 c.cm. is, therefore, capable of protecting contacts for the average duration of the infectivity of the disease and probably longer if they are subjected only to such casual association as occurs in school. But in the home, where the association is much more intimate, passive immunisation does not suffice.

A toxin-antitoxin is now on the market containing 2500 and 10,000 skin test doses of toxin per c.cm., and the dose increases from 0.1 c.cm. to $2 \mathrm{c.cm}$. It is said to confer immunity for about five years. The question is, when will the immunity be conferred to such an extent as will prevent infection from an ordinary kind of exposure? That I do not know. In the immunisation of diphtheria contacts with toxin-antitoxin, three months or more are required, and in these circumstances, in the face of an epidemic, immunisation is valueless for the purposes of that epidemic. Perhaps it may be possible to prolong the passive immunity by repeated injections of antiserum until the active immunity is operating effectively.

The standardising of the dosage of the scarlet fever toxin is still unsatisfactory. The skin test 
dose is unreliable. Okell says that once pyogenic complications of scarlet fever are established antitoxin serum has no effect on them, although the patient may be immune to the toxin. He goes on to say, however, that rabbits may be killed with 5 c.cm. of a young broth culture of the scarlet fever streptococcus given intravenously. Scarlet fever antitoxin which was prepared entirely by immunisation with sterile filtrates (toxins) when given five hours beforehand will prevent the death of the rabbit. Under certain conditions of experiment this effect is quite constant. It is not clear to us if this preventive property of the antitoxin is purely an antitoxin effect, but if it proves to be such it gives us hope that an animal test of an antitoxin activity may be evolved. That raises another point; has the antitoxin any effect in inhibiting the pyogenic complications? I think it has, but the antitoxin I used was prepared by Dochez' method-inoculation of melted agar into the subcutaneous tissues of the horse and implantation thereon of the S. scarlatinoe. It gave rise to multiple abscesses in the horse, and it is possible that any bactericidal effect that I inferred from observation of my cases may have been due to a bacterial immunity provided by the horse serum against the organisms introduced, in addition to the antitoxin against the toxins elaborated from them. My view at the moment is that the best line of treatment for scarlet fever contacts is to provide a passive immunity to cover the first weeks following exposure to infection, and concurrently to confer an active immunity by toxinantitoxin injection.

\section{DIPHTHERIA.}

I suppose it is generally admitted that diphtheria antitoxin is specific in that malady. Accepting that position it must be assumed that the toxin is the cause of the symptoms other than those occurring at the site of the lesion where the organisms are elaborating their poison.

Fortunately, the potency of the antitoxin of diphtheria can be accurately determined. So much is this so that at any particular moment the actual quantity estimated in units can be calculated in a given quantity of blood. It has been ascertained that new-born children possess a certain amount of antitoxin derived from the parent. This diminishes steadily, however, until at about 6 months or so they are unprotected. There are probably some also who have a natural immunity to the disease, but, for the moment, they may be left out of account.

The Schick test is the method employed for determining whether or no there is sufficient antitoxin in the blood to protect against the usual dosage of infection. It is found by Schick's method that immunity is enjoyed by the population as follows :-

$$
\begin{array}{rrrr}
\text { Under } 3 \text { months } . & \ldots & \ldots & 85 \text { per cent. } \\
3 \text { to } 6 \text { months } & . & \ldots & 70 \\
6 \text { months to } 3 \text { years } & \ldots & 40,
\end{array}
$$

That is a passive immunity. Thereafter an active $\frac{0}{0}$ immunity begins to assert itself :-

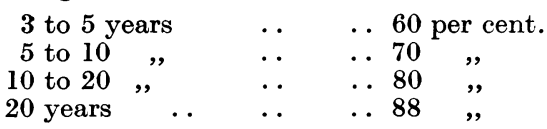

Speaking from memory I think a person is supposed to be immune whose blood containso 1/30 unit of antitoxin per c.cm.

A consideration of these figures indicates that the greatest benefits are likely to be conferred $\widehat{\sigma}$ if active immunity is imparted to the population between the ages of 6 months and 5 years-i.e., in ${ }^{\infty}$ the pre-school age. What is the best way to set? about it? The most scientific way would be to $\overrightarrow{ }$ "Schick" all children at 6 months to 1 year old and immunise the susceptible. That leaveso out those who only enjoy a passive immunity 3 but who will hereafter become susceptible, unlessin they develop an active immunity. There is the N further consideration that Schick reaction is not:always easily and accurately read; and there must be added to that the fact that the Schick test ando its control involve the pricking of the skin twiceand no immunity is conferred as a result.

If we are entitled to assume, as I understand we are, that the toxin-antitoxin causes no ill- $\vec{\bullet}$ effects, would it not be preferable to proceed N at once to immunisation in all cases, and Schick test 3 to 6 months afterwards? We should, ino that case, have the advantage of having partially or wholly immunised the children by the inocula-o tion. It is possible that those pricked twice at $\frac{0}{\Phi}$ the first visit will not return-i.e., if the Schick 2 test is applied-and no good will have been done. $\overrightarrow{\overrightarrow{0}}$ If we can get two, or preferably three inoculations 3 given, we may feel some confidence that the great majority - up to 90 per cent.-are sufficiently? protected.

The unfortunate part of the procedure is that immunity takes three months to develop, so that? as an immediate protection against an epidemic it is of little if any value. The procedure in that event should be to immunise passively by 500 units antitoxin, remove the contact from the infectious agent, and then proceed to immunise actively.

In Aberdeen the procedure is adopted of immunising simultaneously against scarlet fevero and diphtheria. 'The results are awaited with ${ }_{\circ}$ interest. If that system proves effective, and if three injections will confer immunity withouto prejudice to the health of the person beingo immunised, it will obviously be the ideal method of dealing preventively with these two diseases.

It pleases us to record that our President, Sir William Hale-White, and our former chairman $\frac{\vec{P}}{\mathbb{P}}$ of Executive Committee, Sir Humphry Rolleston,, are among those chosen to receive the degree of LL.D. from the University of Edinburgh in this month on the occasion of the annual meeting of the British Medical Association. 